(C) $\{$ Owner/Author $\mid \mathrm{ACM}\}\{2020\}$. This is the author's version of the work. It is posted here for your personal use. Not for redistribution. The definitive Version of Record was published in ACM Digital Library, https://doi.org/10.1145/3401895.3402065 


\section{Communication Cost of Channel Estimation Interpolation for Group-based Vehicular Communications in Cellular Networks}

\author{
Sandra Roger* \\ Computer Science Department \\ Universitat de València \\ 46100 Burjassot, Spain \\ sandra.roger@uv.es \\ Enrique E. Meza-Sánchez \\ Computer Science Department \\ Universitat de València \\ 46100 Burjassot, Spain \\ enmesan@alumni.uv.es
}

\author{
Carmen Botella \\ Computer Science Department \\ Universitat de València \\ 46100 Burjassot, Spain \\ carmen.botella@uv.es \\ Juan J. Pérez-Solano \\ Computer Science Department \\ Universitat de València \\ 46100 Burjassot, Spain \\ juan.j.perez@uv.es
}

\begin{abstract}
Wireless communications for vehicular applications in fifth generation cellular systems (5G) are required to be of low latency and high reliability. Among other factors, the amount of control information to be exchanged between each vehicle and the base station can penalize the communication latency. Several $5 \mathrm{G}$ vehicular use cases involve communications within groups of vehicles, for instance, vehicle platooning. This work is focused on exploiting the structure and characteristics of this particular group-based vehicular service to decrease the control information exchange related to the channel estimation stage necessary for vehicle to infrastructure cellular communications. A scheme based on channel spatial interpolation is proposed, where the real channel is only available at a subset of vehicles, and subsequent spatial interpolation of the channel provides estimates of the large-scale channel parameters for the rest of vehicles in the group. In the paper, communication cost expressions are derived for centralized and distributed topologies, considering a scenario with a macro base station serving a vehicle platoon. The evaluation results show that the distributed topology is more efficient in terms of communication cost, while the centralized architecture is more robust against inter-vehicle distance variations.
\end{abstract}

\section{CCS CONCEPTS}

- General and reference $\rightarrow$ Estimation; • Networks $\rightarrow$ Wireless access points, base stations and infrastructure.

${ }^{*}$ Corresponding author.

Permission to make digital or hard copies of all or part of this work for personal or classroom use is granted without fee provided that copies are not made or distributed for profit or commercial advantage and that copies bear this notice and the full citation on the first page. Copyrights for components of this work owned by others than ACM must be honored. Abstracting with credit is permitted. To copy otherwise, or republish, to post on servers or to redistribute to lists, requires prior specific permission and/or a fee. Request permissions from permissions@acm.org.

EATIS '20, May 13-15, 2020, Aveiro, Portugal

(c) 2020 Association for Computing Machinery.

ACM ISBN X...\$X

https://doi.org/X

\section{KEYWORDS}

Vehicular communications, intelligent transportation systems, channel estimation, spatial interpolation, Kriging, $5 \mathrm{G}$

ACM Reference Format:

Sandra Roger, Carmen Botella, Enrique E. Meza-Sánchez, and Juan J. PérezSolano. 2020. Communication Cost of Channel Estimation Interpolation for Group-based Vehicular Communications in Cellular Networks. In EATIS '20: Euro American Conference on Telematics and Information Systems, May 13-15, 2020, Aveiro, Portugal. ACM, New York, NY, USA, 5 pages. https://doi.org/X

\section{INTRODUCTION}

In the upcoming years, the transport industry is expected to witness an intense period of change and evolution thanks to the implementation of intelligent transportation systems (ITS). These systems rely on wireless communications and new computing and sensor technologies to provide a more efficient, safe and comfortable mobility in a variety of vehicular scenarios (cities, highways, etc.) Probably one of the most interesting features of ITS is the possibility of cooperation between road operators, network infrastructure, vehicles and other road users, increasing the performance that can be achieved by independent systems without cooperation [6]. In particular, the prevention of collisions between vehicles, emergency notification to vehicles, route and track optimization and automated driving are some of the present and future applications of ITS. In order to make all these applications a reality, vehicle to vehicle $(\mathrm{V} 2 \mathrm{~V})$ and vehicle to infrastructure communications (V2I), generally encompassed under the term vehicle to anything (V2X) communication, are fundamental.

In V2X communications, the signal variations caused by mobility often complicate and degrade the channel acquisition task, in the end reducing the system-level performance of e.g. cellular-based alternatives [11]. As a consequence, the transport industry is waiting for the complete definition and rollout of the fifth generation cellular systems (5G) [10] and beyond before implementing the more advanced use cases for ITS, such as autonomous driving. 
It is worth noting that some $5 \mathrm{G}$ vehicular use cases involve groups of users communicating to enable autonomous-driving related services, e.g., to keep the group structure in platooning (grouping of vehicles to travel together [2]), to agree on coordinated maneuvers for cooperative collision avoidance [13] or cooperative lane merging, etc. Related to this, previous works such as [12] have highlighted the spatial correlation of certain wireless-communications measurements in groups of vehicles. More precisely, authors in [12] proposed several schemes intended to reduce the amount of signaling exchanged between groups of communicating vehicles and the base station (BS), focusing on the channel busy ratio or beam information. The main idea was to carry out the measurement reporting from only a subset of vehicles in the group taking into account redundant or correlated information, thus reducing the total overhead.

In this work, we assume that spatial interpolation techniques are used to perform the channel estimation stage in a group of vehicles. More specifically, the minimum number of real channel measurements are obtained from selected vehicles in the group, and Ordinary Kriging (OK) spatial interpolation is then applied to estimate the channel values in the remaining vehicles. This approach can reduce in practice the amount of control information exchanged with respect to a scheme where the channels of all the vehicles are estimated independently. Considering this, we focus on assessing the communication cost reduction achieved in two topologies with a different processing type each, namely centralized and distributed. The communication costs expressions are derived and evaluated in an example scenario, and meaningful insights are obtained towards establishing a reduced signaling protocol for vehicular group-based use cases.

The remaining of the paper is organized as follows. Section 2 introduces the OK spatial interpolation and the communication cost definition. Section 3 states the group-based channel interpolation approach proposed in this paper, and presents the communication cost analysis for the centralized and distributed topologies. Finally, Section 4 evaluates the communication cost expressions and Section 5 draws the conclusions and indicates future work.

\section{SYSTEM MODEL}

\subsection{Scenario}

Figure 1 depicts the scenario considered in this paper. We target the particular use case of a $\mathrm{N}$-vehicle platoon (each vehicle of length l) passing through a BS assisting the platooning communication service. The BS is aligned with the middle point of the platoon in the direction of the road and located at a distance $R$ from the road border. All vehicles are assumed to be perfectly aligned and separated of each other a distance equal to $L$, known as platoon inter-vehicle distance. Alignment and $L$ will be in practice guaranteed by a suitable platooning communication protocol. To simplify the derivations, vehicles are further assumed to be driving on the centre of the lane. Note that small deviations from the center (upper bounded by $w / 2$ ) would have a minor impact on the distance from each vehicle to the BS, due to the fact that $R$ is an order of magnitude higher than $w / 2$. Regarding the vehicle antennas, these are located in the middle of the roof, assuming for simplicity that their position matches the middle point of the vehicle in both directions.

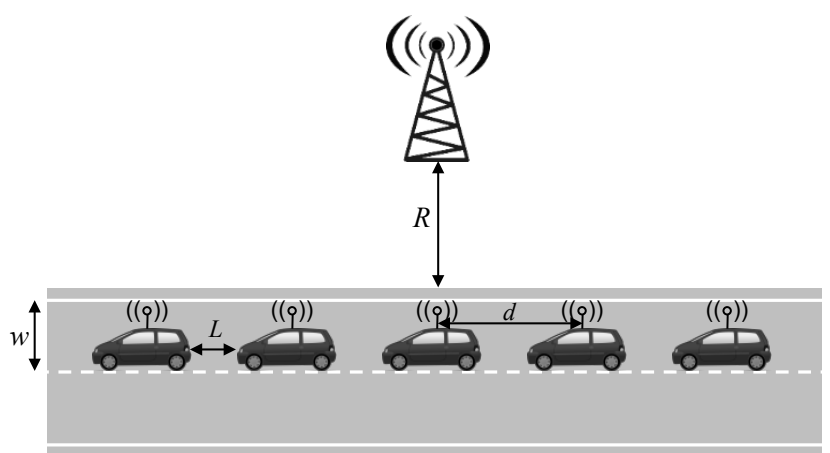

Figure 1: Considered scenario. Platoon of $N$ vehicles served by a BS.

As a result, the antennas of two consecutive vehicles are separated a distance equal to $d=L+l$. Lane width is denoted by $w$.

As stated in the introduction, we consider that OK is applied in this setting to estimate the channel in the $N$-vehicle platoon, starting from a subset of $P$ known channel values. Note that spatial interpolation methods have recently gained interest in $5 \mathrm{G}$ automotive applications due to their ability to reconstruct the radio environment map (REM) of a given BS from a subset of available measurements (see [5][4] and references therein). The minimum number of channel values, $P$, are assumed to be estimated at certain vehicles, and then the OK method is applied to perform channel interpolation and to obtain the estimated channel values for rest of the platoon vehicles. Theoretically, the range of transmission for each vehicle is limited, but we assume that the distance $d$ fulfills this value, and that multi-hop communication is possible to communicate between non-adjacent platoon vehicles. The details of the OK technique are described in what follows.

\subsection{Ordinary Kriging spatial interpolation}

Let us assume that the field value measured by a vehicle located at position $\mathbf{x}_{i}$ is denoted by $V\left(\mathbf{x}_{i}\right), i=\{1,2, \ldots, N\}$. In our case, the complete REM is not needed, the target locations are narrowed down to the $N$ vehicles positions in the platoon, and the known field locations are a subset of $P$. The estimated field value is denoted by $\hat{V}\left(\mathbf{x}_{0}\right)$ at locations $\mathbf{x}_{0}$ where the field is unknown (remaining $N-P$ vehicles in our case).

Two steps are required in OK interpolation, semivariogram analysis (measures the correlation between field samples) and Kriging prediction. The empirical semivariogram $(\mathrm{EV}), \hat{\gamma}(\mathbf{h})$, is defined as follows:

$$
\hat{\gamma}(\mathbf{h})=\frac{1}{2|\mathcal{N}(\mathbf{h})|} \sum_{\mathcal{N}(\mathbf{h})}\left(V\left(\mathbf{x}_{i}\right)-V\left(\mathbf{x}_{j}\right)\right)^{2},
$$

where $\mathbf{h}=\mathbf{x}_{i}-\mathbf{x}_{j}$ is the lag distance, and $V\left(\mathbf{x}_{i}\right)$ and $V\left(\mathbf{x}_{j}\right)$ are field values at spatial locations $\mathbf{x}_{i}$ and $\mathbf{x}_{j}$, respectively. $\mathcal{N}(\mathbf{h})$ is the set comprising all location pairs $\left(\mathbf{x}_{i}, \mathbf{x}_{j}\right)$ such that $\mathbf{x}_{i}-\mathbf{x}_{j}=\mathbf{h}$ and $|\cdot|$ denotes its cardinality. OK replaces the EV by a semivariogram model, which in this case is the spherical model given by expression:

$$
\bar{\gamma}(\mathbf{h})=\left\{c_{1}+c_{2}\left\{\frac{3}{2}\left(\frac{\mathbf{h}}{c_{3}}\right)-\frac{1}{2}\left(\frac{\mathbf{h}}{c_{3}}\right)^{3}\right\},\right.
$$


where $c_{1}, c_{2}$ and $c_{3}$ are nugget, sill and range variables, respectively [? ]. In a second step, ordinary Kriging prediction is performed, where the Kriging interpolator at target location $\mathbf{x}_{0}$ is given by:

$$
\left.\hat{V}\left(\mathbf{x}_{0}\right)\right|_{P}=\sum_{i=1}^{P} w_{i \mid P}\left(\mathbf{x}_{0}\right) V\left(\mathbf{x}_{i}\right),
$$

where $P$ is the number of nodes whose estimates are used to perform the prediction, $w_{i \mid P}$ is the weight assigned for node $i$ from an estimation performed using $P$ nodes and $\left.\hat{V}\left(\mathbf{x}_{0}\right)\right|_{P}$ is the estimated value. These weights can be efficiently solved by rewriting the equations into a matrix system [? ]. Interestingly, vehicles are equipped with GPS, which largely simplifies the calculation of the distances involved in the semivariogram model evaluation.

In this work, we focus on acquiring channel estimates, thus considering that the field value at location $\mathbf{x}_{i}$ is given by:

$$
V\left(\mathbf{x}_{i}\right)=P\left(\mathbf{x}_{i}\right)+S\left(\mathbf{x}_{i}\right),
$$

where $P\left(\mathbf{x}_{i}\right)$ is the average received power depending on the path loss model and $S\left(\mathbf{x}_{i}\right)$ is the shadow fading following a lognormal distribution [8]. The received power $(\mathrm{dBm})$ at location $\mathbf{x}_{i}$ from a single antenna BS is calculated by the simple path-loss model:

$$
P\left(\mathbf{x}_{i}\right)=K_{d B}+10 \alpha \log _{10} d_{0}+10 \log _{10} d_{i}^{-\alpha},
$$

where $K_{d B}$ is the constant path loss factor in $\mathrm{dB}$ units, $\alpha$ is the path loss exponent, $d_{0}$ is a reference distance for antenna far field and $d_{i}$ is the distance between the vehicle location $\mathbf{x}_{i}$ and the BS location.

To improve the field value estimation, authors in [3] proposed a novel distributed clustering algorithm to perform the channel estimation using subsets of measurements or clusters of nodes for OK, that were optimized by using the Kriging variance as a formation parameter. Basically, an initial cluster with a minimum number of $P$ nodes was built with the nodes that were located closest to the target location $\mathbf{x}_{0}$, and new nodes were added one by one as long as the Kriging variance decreased, thus enhancing the quality of the obtained field value estimation at $\mathbf{x}_{0}$. In [7], this algorithm was modified to consider the communication cost as the fundamental parameter for the clustering formation algorithm in the OK framework. Note that, in a general setting, a set of possibly overlapping clusters was obtained, since a different cluster of nodes was formed for each one of the unknown positions $\mathbf{x}_{0}$. Since in this work we consider ITS use cases involving groups of vehicles communicating in close proximity, the vehicular service group can be naturally exploited as the estimation cluster, simplifying the cluster formation task. In our scheme, we shift the cluster paradigm and we focus instead on evaluating the communication cost reduction achieved when using a minimum number of real channel measurements from closest vehicles to estimate or predict the channel value in the remaining vehicles of the group or cluster.

\subsection{Communication cost modeling}

Communication cost has been largely researched in the literature of wireless sensor networks [7, 9, 14]. Models for this cost rely on several parameters such as the energy dissipation required to communicate between nodes, the data rate and packet size in bits, the distance between the nodes, or the channel model parameters (mainly the path loss exponent). In [7], several communication cost models were analyzed towards establishing a suitable option in the case of $\mathrm{OK}$ interpolation. In this work, we use the cost function proposed in $[7,9,14]$, given as follows:

$$
f_{c}=\frac{N_{0}\left(2^{B}-1\right) \mathrm{SNR}}{\mu g_{i}},
$$

where $g_{i}=x_{i} d_{i}^{-\alpha}$ is the channel gain between vehicle $i$ and the BS and $x_{i}$ is a constant chosen randomly following an exponential distribution with mean equal to one. The total number of bits is defined by B. $\mu(\mu>0)$ is a parameter that depends on the particular modulation scheme. SNR is the desired Signal-to-Noise ratio, while $N_{0}$ is the power spectral density of the noise.

In [7], the model in Equation (5) was simplified and the impact of the packet size in bits was neglected. In our case, the packet size in bits will be fundamental to evaluate the communication cost of the proposed topologies. However, we assume that parameters related to the modulation schemes such as $\mu$, as well as $N_{0}$ and the SNR are common for the group and can be disregarded within the cost comparison without loss of generality.

\section{GROUP-BASED CHANNEL ESTIMATION INTERPOLATION}

As described in the previous section, the OK technique requires a set of field values to provide an estimation at locations where the field is unknown. In our case, we assume a minimum of $P$ available channel values, whereas the estimation is performed for the remaining $N-P$ vehicle locations. For the purpose of channel estimation, we focus on two different topologies, a centralized one, where the vehicles performing the channel estimation forward their measurements to the BS for computing Equation (2) in a centralized setting, and a distributed one, where the estimation in (2) is directly calculated at the remaining $N-P$ vehicles by message passing among the vehicles in the group. From a communication point of view, we consider an ideal system, i.e. without communication errors nor message re-transmissions. Given that in this case the OK channel interpolation scheme will lead to the same estimated channel values in both settings, we focus on comparing only the communication cost.

\subsection{Centralized versus distributed approach}

Figures 2 (a) and (b) show the message exchange for a centralized and distributed channel estimation, respectively, in the vehicle platooning scenario of interest. Since, following the derivations of [4], a minimum of $P=3$ channel measurements are required for performing OK channel estimation interpolation, in Figure 2 we assume that vehicles 1, 3 and 5 have true channel values, while channel estimates are needed for vehicles 2 and 4 . Note that the initial step of acquiring the channels between the BS and vehicles 1, 3 and 5 using pilot transmissions is common to both topologies, and it has been intentionally omitted in the figure and in the analysis for simplicity.

When the centralized approach is considered, the BS receives in a first step the $P=3$ true channel values for the channel interpolation task (represented by solid arrows). After the interpolation scheme is applied, the $\mathrm{BS}$ reports the estimated $N-P$ channel values to the vehicles of interest. In the example, the number of estimates is 


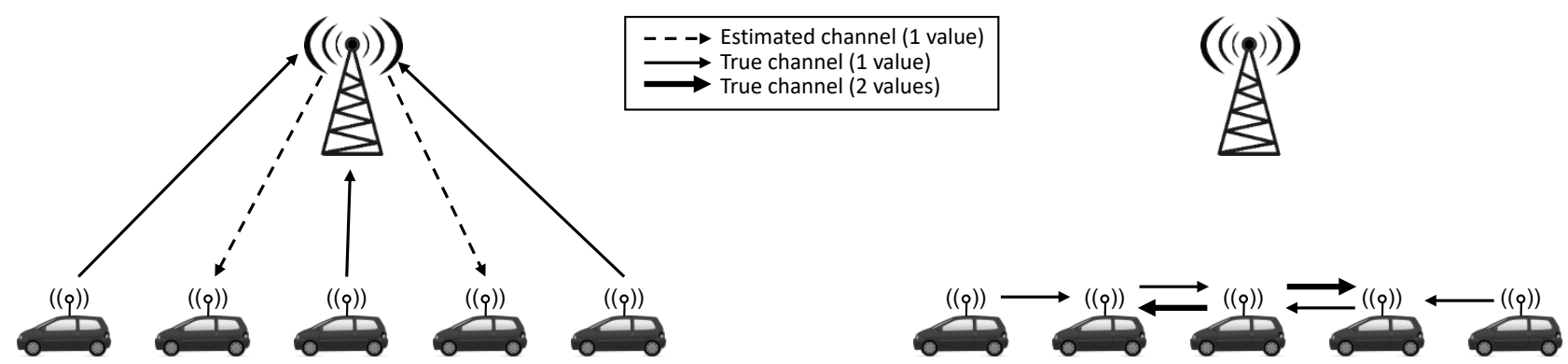

(a)

(b)

Figure 2: Proposed channel estimation schemes in a vehicular platoon. (a) Centralized, (b) Distributed. Vehicles 1, 3 and 5 have true channel values, while channel estimates are needed for vehicles 2 and 4.

just 2 channel values (dashed arrows). As a result, the number of exchanged messages equals $N$.

In the distributed scheme, channel interpolation is carried out directly at the vehicles with again at least $P=3$ true channel values received by message passing from other vehicles in the group. As already mentioned, for simplicity we consider that each vehicle gets the closest available channel values, thus minimizing the number of exchanged messages. Considering the example in Figure 2 (b), vehicle 2 would receive the true channels of vehicles 1 and 3 from two direct messages, and the true channel of vehicle 5 from a multihop transmission through vehicles 4 and 3. For efficiency, vehicle 3 is assumed to report two true channel values embedded in the same message (the one of vehicle 5 together with its own). Messages carrying two channel values are represented using thicker solid arrows than those carrying only one. A similar approach is followed to provide the three true channel values to vehicle 4 . In a general case, the message exchange will involve $N-1$ messages with one channel value and $N-P$ messages with two channel values.

\subsection{Communication cost analysis}

For the centralized approach, assuming that the BS is located in the origin of coordinates $(0,0)$, the Euclidean distance between the BS and platoon vehicle number $n$ can be calculated as follows (see Figures 1 and 2(a)):

$$
d_{n}=\sqrt{\left(R+\frac{w}{2}\right)^{2}+d^{2} \cdot\left(n-\left(\frac{N-1}{2}+1\right)\right)^{2}},
$$

where the values of $n$ range from 1 for the platoon leader to $N$ for the last platoon member.

Once the distances for all vehicles are obtained, the communication cost for the centralized estimation can be calculated according to Equation (5) as:

$$
\operatorname{Cost}_{\mathrm{C}}=\sum_{n=1}^{N}\left(\frac{2^{B}-1}{d_{n}^{-\alpha}}\right),
$$

where the $\mu$ factor has been simplified to consider only the distance contribution, and parameters related to the modulation scheme, as well as $N_{0}$ and the SNR are common to all the vehicles.
In the distributed approach, according to Figure 2(b), all the messages are transmitted though one-hop communications between neighboring vehicles. As a result, all the messages traverse exactly the same distance, equal to the separation between antennas of consecutive vehicles $(d)$. However, recall that in some cases the messages carry true channel values from two vehicles, which has been reflected in the cost calculations by doubling the number of bits. The estimated cost results in:

$$
\operatorname{Cost}_{\mathrm{D}}=(N-1)\left(\frac{2^{B}-1}{d^{-\alpha}}\right)+(N-P)\left(\frac{2^{2 B}-1}{d^{-\alpha}}\right),
$$

where the first term comes from the transmission of messages with a single channel value and the second term from the messages with two channel values.

\section{PERFORMANCE RESULTS}

We focus on a snapshot of the platoon service communication, where the vehicles are static. As an example, the parameters of a 3GPP-like freeway scenario are considered, where the BS is located $R=35 \mathrm{~m}$ away from the road border and each lane has a width $w=4 \mathrm{~m}$ [1]. Two particular cases for the inter-vehicle distance are evaluated: $L=2 \mathrm{~m}$ (high-density platooning) and $L=10 \mathrm{~m}$ (normal platooning). A typical vehicle length of $l=4.7 \mathrm{~m}$ is assumed. The particular values for the path loss parameters in Equation (4) are extracted directly from [1]. More specifically, path loss exponent takes the value $\alpha=3.7$, the reference distance for antenna far field is $d_{0}=1 \mathrm{~km}$, and the constant path loss factor at $d_{i}=d_{0}$ is $K_{d B}=137 \mathrm{~dB}$.

Evaluations of the derived cost expressions in Equations (7) and (8) were carried out using MATLAB, where the number of platoon vehicles $N$ was varied from 4 to 10 , considering in all cases that the minimum of $P=3$ vehicles knew the true channel to carry out the interpolation. Figure 3 shows the estimated cost of the centralized and distributed approaches in the considered example scenario, applying Equations (7) and (8), respectively. In these evaluations the parameter $B$ is set to 1 for simplicity, since its effect contributes equally to all the evaluations. It can be observed that, despite the fact that the distributed approach exchanges a larger number of messages (23 messages for $N=10$ ) the communication cost is substantially lower. This is due to the higher proximity between the 


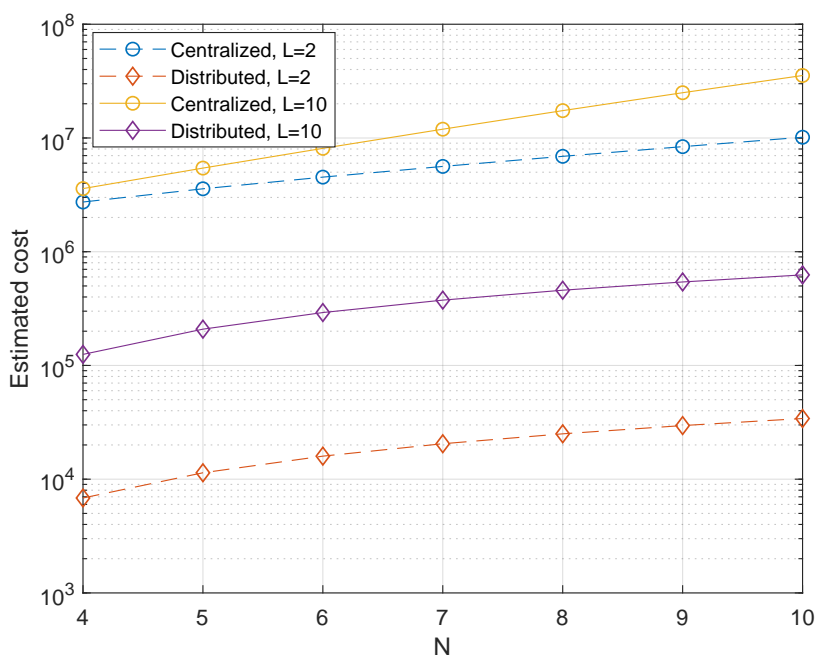

Figure 3: Estimated cost versus number of vehicles for the centralized and distributed channel estimation approaches with $L=2 \mathbf{m}$ and $L=10 \mathbf{m}$.

communication points, which increases the denominator term in the cost functions. The centralized approach shows a linear increase, whereas the distributed scheme increases in a logarithmic fashion.

Regarding the effect of the inter-vehicle distance $(L)$, the results show that it has a higher impact on the distributed scheme, showing a constant cost gap of more than an order of magnitude for all numbers of vehicles. This result is reasonable, as the distance used for the cost estimation is straightforwardly related to this parameter. However, in the centralized scheme, the communication cost gap is not constant and increases linearly with the number of vehicles. Recall that $L$ does not contribute to the cost of the centralized scheme directly, but combined with a term that includes also the distance from the BS to the road border $R$ (see Equation (6)). Therefore, its contribution turns more significant as its magnitude approaches the one of $R$. The latter effect also justifies that the cost gap between centralized and distributed approaches decreases as $L$ gets higher for a given $N$.

\section{CONCLUSION}

This work proposed the use of Ordinary Kriging spatial interpolation to reduce the control information dedicated to the channel estimation stage in group-based vehicular communications. Assuming that the real channel is only acquired by a subset of vehicles, spatial interpolation of the channel provides estimates of the large-scale channel parameters for the rest of vehicles in the group. Communication cost expressions have been derived for two alternatives, a centralized approach and a distributed one, considering a communication scenario between a macro base station and a vehicle platoon. The evaluation results show that the distributed topology is more efficient in terms of communication cost, while the centralized scheme is more robust against inter-vehicle distance variations.
As a future work, a more complete performance evaluation of the proposed interpolation-based channel estimation in terms of channel accuracy and feedback overhead should be carried out, where both parameters will be in trade-off.

\section{ACKNOWLEDGMENTS}

The work of Dr. Sandra Roger was supported by the Spanish Ministry of Science, Innovation and Universities under Grant RYC2017-22101. Dr. Carmen Botella would like to thank the support of the University of Valencia (Vicerectorat d'Ocupació i Programes Formatius) through the project UV-SFPIE PID19-1097673.

\section{REFERENCES}

[1] 3GPP TSG RAN. 2016. Study on LTE-based V2X services. TR 36.885 V14.0.0. 3GPP.

[2] C. Campolo, A. Molinaro, G. Araniti, and A. O. Berthet. 2017. Better Platooning Control Toward Autonomous Driving : An LTE Device-to-Device Communications Strategy That Meets Ultralow Latency Requirements. IEEE Vehicular Technology Magazine 12, 1 (March 2017), 30-38.

[3] V. Chowdappa, C. Botella, and B. Beferull-Lozano. 2015. Distributed Clustering Algorithm for Spatial Field Reconstruction in Wireless Sensor Networks. In 2015 IEEE 81st Vehicular Technology Conference (VTC Spring). 1-6.

[4] V. Chowdappa, C. Botella, J. J. Samper-Zapater, and R. J. Martinez. 2018. Distributed Radio Map Reconstruction for $5 \mathrm{G}$ Automotive. IEEE Intelligent Transportation Systems Magazine 10, 2 (Summer 2018), 36-49.

[5] E. Dall'Anese, S.J. Kim, and G.B. Giannakis. 2011. Channel Gain Map Tracking via Distributed Kriging. IEEE Transactions on Vehicular Technology 60, 3 (Feb 2011), 1205-1211.

[6] ETSI. 2013. Intelligent Transport Systems (ITS); Cooperative ITS (C-ITS). TR 101 607 V1.1.1. ETSI

[7] F. Frantzis, V. Chowdappa, C. Botella, J. J. Samper, and R. J. Martinez. 2017. Radio Environment Map Estimation Based on Communication Cost Modeling for Heterogeneous Networks. In 2017 IEEE 85th Vehicular Technology Conference (VTC Spring). 1-6.

[8] A. Goldsmith. 2005. Wireless Communications. Cambridge University Press.

[9] Y. Huang and Y. Hua. 2009. Energy Planning for Progressive Estimation in Multihop Sensor Networks. IEEE Transactions on Signal Processing 57, 10 (Oct 2009), 4052-4065.

[10] P. Marsch, O. Bulakci, O. Queseth, and M. Boldi. 2018. 5G System Design: Architectural and Functional Considerations and Long Term Research. Wiley.

[11] D. Martín-Sacristán, S. Roger, D. Garcia-Roger, J. F. Monserrat, A. Kousaridas, P. Spapis, S. Ayaz, and C. Zhou. 2018. Evaluation of LTE-Advanced Connectivity Options for the Provisioning of V2X Services. In IEEE Wireless Communications and Networking Conference (WCNC). Barcelona, Spain.

[12] D. Martín-Sacristán, S. Roger, D. Garcia-Roger, J. F. Monserrat, A. Kousaridas, P. Spapis, and C. Zhou. 2019. Signaling Reduction in 5G eV2X Communications Based on Vehicle Grouping. In 2019 European Conference on Networks and Communications (EuCNC). 427-431.

[13] S. Roger, D. Martín-Sacristán, D. Garcia-Roger, J. F. Monserrat, P. Spapis, A. Kousaridas, S. Ayaz, and A. Kaloxylos. 2019. Low-Latency Layer-2-Based Multicast Scheme for Localized V2X Communications. IEEE Transactions on Intelligent Transportation Systems 20, 8 (Aug. 2019), 2962-2975.

[14] S. Shah and B. Beferull-Lozano. 2013. Joint Sensor Selection and Multihop Routing for Distributed Estimation in Ad-hoc Wireless Sensor Networks. IEEE Transactions on Signal Processing 61, 24 (Dec 2013), 6355-6370. 